\title{
Models of infrared spectra of Sakurai's Object (V4334 Sgr) in $1997^{\star}$
}

\author{
Y. V. Pavlenko ${ }^{1,2}$ and T. R. Geballe ${ }^{3}$ \\ 1 Main Astronomical Observatory of Academy of Sciences of Ukraine, Golosiiv woods, Kyiv-127, 03680 Ukraine \\ 2 Isaac Newton Institute of Chile, Kiev Branch \\ 3 Gemini Observatory, 670 N. A'ohoku Place, Hilo, HI 96720 USA \\ e-mail: tgeballe@gemini.edu
}

Received 5 March 2002 / Accepted 17 May 2002

\begin{abstract}
Theoretical spectral energy distributions computed for a grid of hydrogen-deficient and carbon-rich model atmospheres have been compared with the observed infrared (1-2.5 $\mu \mathrm{m})$ spectra of V4334 Sgr (Sakurai's Object) on 1997 April 21 and July 13. The comparison yields an effective temperature of $T_{\text {eff }}=5500 \pm 200 \mathrm{~K}$ for the April date and $T_{\text {eff }}=5250 \pm 200 \mathrm{~K}$ for July. The observed spectra are well fitted by Asplund et al. (1999) abundances, except that the carbon abundance is higher by 0.3 dex. Hot dust produces significant excess continuum at the long wavelength ends of the 1997 spectra.
\end{abstract}

Key words. stars: individual: V4334 Sgr (Sakurai's Object) - stars: AGB and post-AGB - stars: atmospheres stars: fundamental parameters

\section{Introduction}

V4334 Sgr (Sakurai's Object), the "novalike object in Sagittarius" discovered by Y. Sakurai on February 20, 1996 (Nakano et al. 1996) is a very rare example of extremely fast evolution of a star during a very late final helium-burning event (Duerbeck \& Benetti 1996). During the first few months after discovery, Sakurai's Object increased in visual brightness to $V \sim 12^{\mathrm{m}}$. In 1997 it increased further to $V \sim 11^{\mathrm{m}}$. In March 1997 the first evidence of dust formation was seen (Kimeswenger et al. 1997; Kamath \& Ashok 1999; Kerber et al. 2000). In early 1998 the optical brightness of Sakurai's Object decreased (dimming first reported by Liller et al. 1998), but then recovered. However, during the second half of 1998 an avalanche-like growth of the dusty envelope occurred, causing a rapid decrease in optical brightness and the complete visual disappearance of the star in 1999. At present essentially only thermal emission by dust can be observed (Geballe et al. 2002). Our view of the born again star has been completely obscurred by the dust it has produced.

Abundance analyses by Asplund et al. (1997, 1999) and Kipper \& Klochkova (1997) have found peculiarities similar to

Send offprint requests to: Ya. V. Pavlenko,

e-mail: yp@mao.kiev .ua

* Based on observations obtained at the United Kingdom Infrared Telescope (UKIRT), which is operated by the Joint Astronomy Centre on behalf of the U. K. Particle Physics and Astronomy Research Council. those of R CrB-like stars. Asplund et al. (1999) estimate that the logarithmic abundances of hydrogen, helium and carbon in atmosphere of Sakurai's Object in October 1996 were -2.42, -0.02 and -1.62 , respectively ${ }^{1}$, with hydrogen only the third most abundant element by number. All of the above studies are based on optical spectra obtained in 1996. At that time the spectrum of Sakurai's Object resembled that of an F-supergiant; molecular bands were absent or very weak. Cooling of the photosphere of Sakurai's Object resulted in its optical spectrum during 1997 and 1998 resembling those of C-giants with very strong bands of $\mathrm{CN}$ and $\mathrm{C}_{2}$ (Pavlenko et al. 2000). Modeling of some of these optical spectra have allowed estimates of the changes in $T_{\text {eff }}$ and $E_{B-V}$ to be made during this period of rapid evolution of the optical spectrum (Pavlenko et al. 2000; Pavlenko \& Duerbeck 2001).

Modeling of near infrared (1-2.5 $\mu \mathrm{m})$ spectra of Sakurai's Object is of interest for several reasons. In addition to providing comparisons with results obtained from the optical spectrum and tests of the reliability of molecular and atomic data, it allows accurate determination of the effective temperature and sensitive tests for emission by hot dust. Use of the 1-2.5 $\mu \mathrm{m}$ region for modeling is especially important after 1996, when the bulk of the photospheric flux shifted from the optical into this waveband.

In this paper we present and compare model $1-2.5 \mu \mathrm{m}$ spectra with those of Sakurai's Object obtained during 1997, on UT

\footnotetext{
1 In this work we use an abundance scale $\sum N_{i}=1$.
} 
April 21 and July 13 at the United Kingdom Infrared Telescope (UKIRT). The observed spectra together with observational details were presented by Eyres et al. (1998) and the July spectrum is also shown in Geballe et al. (2002). The resolutions of these spectra as presented here are $1.4 \mathrm{~nm}(0.0014 \mu \mathrm{m})$ at $1.02-$ $1.35 \mu \mathrm{m}$ and $2.8 \mathrm{~nm}(0.0028 \mu \mathrm{m})$ at $1.42-2.52 \mu \mathrm{m}$. Narrow spectral features in the $1.82-1.95 \mu \mathrm{m}$ portions of these spectra are due to incomplete removal of strong telluric lines.

\section{Modeling procedure}

Grids of plane-parallel model atmospheres in LTE, with no energy divergence were computed by the SAM12 program (Pavlenko 2002). This program is a modification of ATLAS12 (Kurucz 1999). Opacities due to C I bound-free absorption, of importance in atmospheres of hydrogen-deficient, carbonrich stars over a wide $(0.1-8 \mu \mathrm{m})$ wavelength region (see Pavlenko 1999, 2002; Asplund et al. 2000), were computed using the OPACITY PROJECT cross sections database (Seaton et al. 1992). The opacity of $\mathrm{C}^{-}$also was taken into account Myerscough \& McDowell 1996; see Pavlenko 1999 for more details).

An opacity sampling approach (Sneden et al. 1976) was used to account for atomic and molecular line absorption. The source of the atomic line information was the VALD database (Kupka et al. 1999). Lists of diatomic molecular lines of ${ }^{12} \mathrm{CN}$, ${ }^{13} \mathrm{CN},{ }^{12} \mathrm{C}_{2},{ }^{13} \mathrm{C}_{2},{ }^{12} \mathrm{C}^{13} \mathrm{C},{ }^{12} \mathrm{CO},{ }^{13} \mathrm{CO}, \mathrm{SiH}$, and $\mathrm{MgH}$ were taken from Kurucz (1993). We adopted Voigt profiles for every absorption line; damping constants were computed following Unsold (1955). Microturbulent velocities of 3-6 $\mathrm{km} \mathrm{s}^{-1}$ were adopted.

Two grids of model atmospheres with different abundances were computed. One grid used the chemical composition of Sakurai's Object determined by Asplund et al. (1999) for October 1996. The other is the same except that the abundance of carbon is increased by 0.6 dex. This "carbon-rich" case is of interest because the carbon abundance of Asplund et al. (1997) is larger by 0.6 dex than that which was obtained by them later from the analysis of high resolution spectra (see Asplund et al. 1997, 1999, 2000. This "carbon problem" appears to arise more from the analysis of C I lines than from C II or $\mathrm{C}_{2}$ lines (Asplund, private communication). For both grids the isotopic ratio ${ }^{12} \mathrm{C} /{ }^{13} \mathrm{C}=5$ was adopted (Asplund et al. 1997).

To determine molecular densities, a system of equations of chemical equilibrium was solved for a mixture of $\sim 70$ atoms, ions and molecules, including the most abundant diatomic molecules containing carbon. We used the approach developed by Kurucz in ATLAS12 (Kurucz 1999) in which ratios of densities of atoms and $n_{x}, \ldots, n_{z}$ and molecules $n_{x y \ldots z}$ follow the equations:

$$
\begin{aligned}
n_{x} * \ldots * n_{z} / n_{x \ldots z}=\exp \left(-D_{0} / T_{\mathrm{ev}}+b\right. \\
\\
-c *(T+d *(T-e *(T+f * T))) \\
+3 / 2 *(m-k-1) * \ln T),
\end{aligned}
$$

where $D_{0}$ and $T_{\mathrm{ev}}$ are the dissociation potential and the temperature in $\mathrm{eV}$ (for neutral diatomic molecules $m=1, k=0$ ). The adopted molecular constants for the three most important molecules are given in Table 1.
Synthetic spectra ${ }^{2}$ were computed using the WITA6 program (Pavlenko 1999) for the same grid of opacities, abundances, isotopic ratios and microturbulent velocites that were adopted for the model atmospheres. WITA6 computes spectra and spectral energy distributions (SEDs) taking into account "line by line" absorption by atomic and molecular transitions. For Sakurai's Object spectra computed with wavelength step $0.05 \mathrm{~nm}$ were convolved with gaussian profiles with full widths at half maximum of 1.4 and $2.8 \mathrm{~nm}$ over the appropriate wavelength intervals. Computed and observed spectra were normalized at $1.7 \mu \mathrm{m}$ for comparison; see Pavlenko et al. (2000) for more details.

\section{Results}

\subsection{Principal spectral features}

In Fig. 1 the principal features formed by the molecular species $\mathrm{C}_{2}, \mathrm{CO}$, and $\mathrm{CN}$ are displayed as separate spectra. Atomic features are also shown, as these are also present in Sakurai's Object (see Eyres et al. 1998; Geballe et al. 2002). As in the optical spectrum (Pavlenko et al. 2000), absorption of only a few molecular species accounts for the main features in the IR spectrum. Only the most abundant isotopic species of each molecule is shown. Of the less abundant isotopic species, only bands of ${ }^{13} \mathrm{CO}$ have been detected in the infrared (Eyres et al. 1998).

\subsection{Dependences on $T_{\text {eff }}, \log g, V_{t}$ and $\log N(H)$}

The model spectra of Sakurai's Object display a strong dependence on $T_{\text {eff }}$ (Fig. 2). ${ }^{3}$ In general, the dependence of the IR SED on $T_{\text {eff }}$ is determined mainly by the variations of the molecular densities with temperature. The band strengths of $\mathrm{CN}, \mathrm{CO}$ and $\mathrm{C}_{2}$ all increase as $T_{\text {eff }}$ decreases. Changes in the continuum fluxes are much smaller. Similar effects are seen in model optical spectra (Pavlenko \& Yakovina 2000). However, there the molecular bands are numerous, whereas in the infrared only the few strongest vibration-rotation bands of $\mathrm{CN}$, $\mathrm{C}_{2}$, and $\mathrm{CO}$ are prominent.

As can be seen in Fig. 3, the dependence of the spectrum on $\log g$ is generally rather weak. However, there are differences in the responses of different spectral regions. The strong molecular bands show rather weak dependence on $\log g$, whereas the fluxes at $1.25-1.35,1.60-1.75,1.9-2.2$ microns show more noticeable changes.

Previous abundance analyses of the spectra of Sakurai's Object and related $\mathrm{R} \mathrm{CrB}$ stars indicate microturbulent velocities $V_{\mathrm{t}}$ in the range $5-8 \mathrm{~km} \mathrm{~s}^{-1}$ (cf. Asplund et al. 2000). The value of $V_{\mathrm{t}}$ affects the spectral distribution, as is shown in Fig. 4. The effect of $V_{\mathrm{t}}$ on the IR spectra of Sakurai's Object is larger at the heads of molecular bands than elsewhere, because

\footnotetext{
${ }^{2}$ In fact these are spectral energy distributions (SEDs).

${ }^{3}$ For $\lambda>2.7 \mu$ m model spectra shown here and in subsequent plots were computed without molecular absorption; i.e, they are continuum fluxes, which provide information about the dependence of the continuum fluxes on input parameters.
} 
Table 1. Dissociation equilibrium constants of $\mathrm{CO}, \mathrm{C}_{2}$ and $\mathrm{CN}$.

\begin{tabular}{ccccccc}
\hline \hline Molecule & $D_{0}$ & $b$ & $c * 1 . \mathrm{E} 2$ & $d * 1 . \mathrm{E} 6$ & $e * 1 . \mathrm{E} 10$ & $f * 1 . \mathrm{E} 15$ \\
\hline $\mathrm{C}_{2}$ & 6.116 & 48.75 & .2192 & .4149 & .4121 & 1.550 \\
$\mathrm{CN}$ & 7.700 & 47.45 & .1332 & .1989 & .1778 & .6323 \\
$\mathrm{CO}$ & 11.105 & 49.45 & .1651 & .3103 & .3100 & 1.168 \\
\hline
\end{tabular}

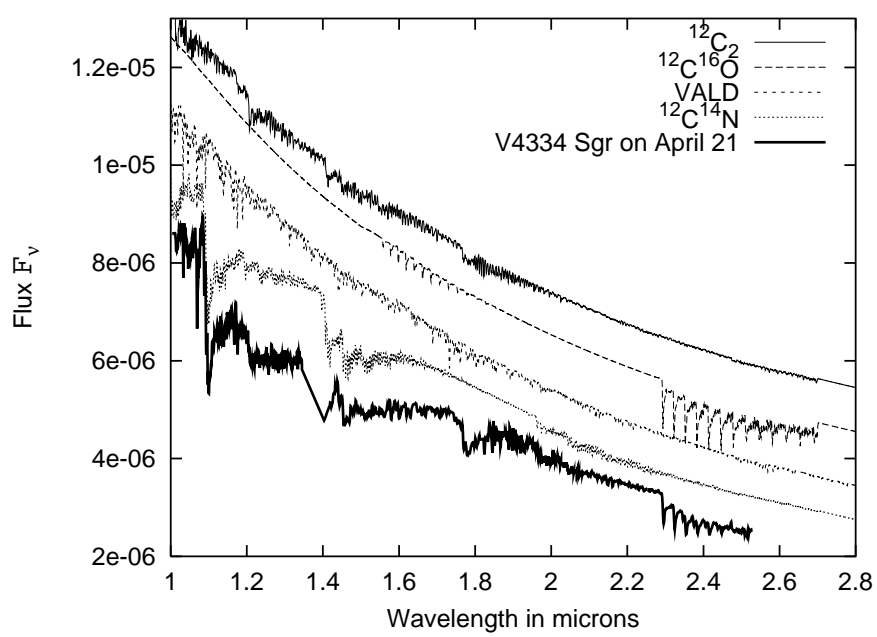

Fig. 1. Model spectra of species that produced the strongest absoption features in the 1.0-2.5 $\mu \mathrm{m}$ spectrum of Sakurai's Object spectra during 1997, computed for $T_{\text {eff }} / \log g=5500 / 0.0$ model atmosphere with Asplund et al. (1999) abundances for October 1996. The model spectrum due to atomic species alone, labelled VALD (see text) is also shown. Spectra are artificially shifted on the $y$-axis.

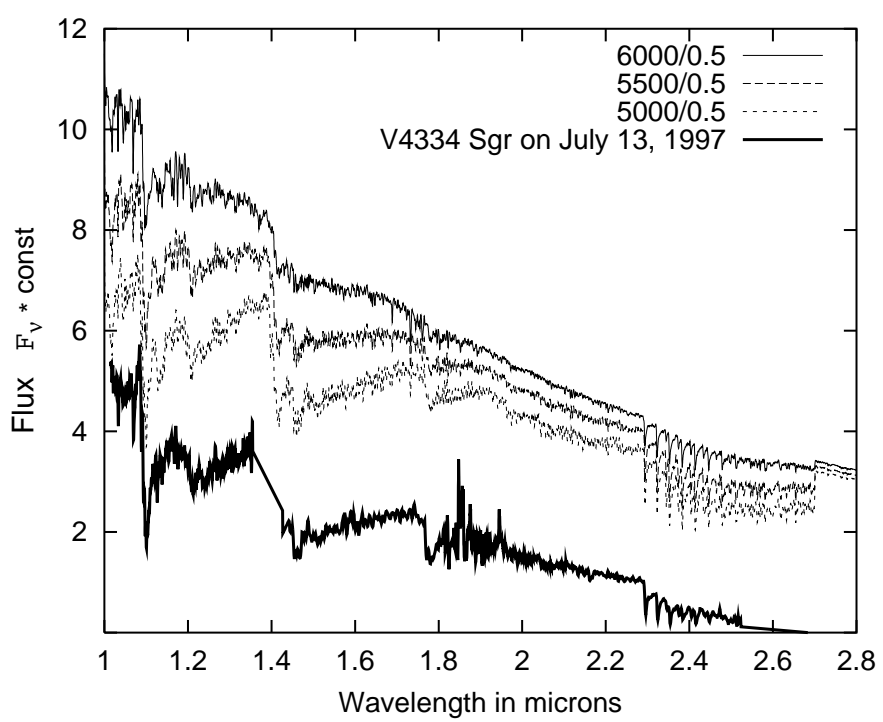

Fig. 2. Dependence of the model IR spectrum on $T_{\text {eff }}$. The model spectra use Asplund et al. (1999) abundances for October 1996. The observed spectrum of Sakurai's Object on July 13, 1997 is shifted on the $y$-axis.

the heads are formed by closely packed molecular lines whose overall absorption is sensitive to $V_{\mathrm{t}}$.

The main sources of line opacity in the model atmospheres approximating Sakurai's Object in 1997 are molecular

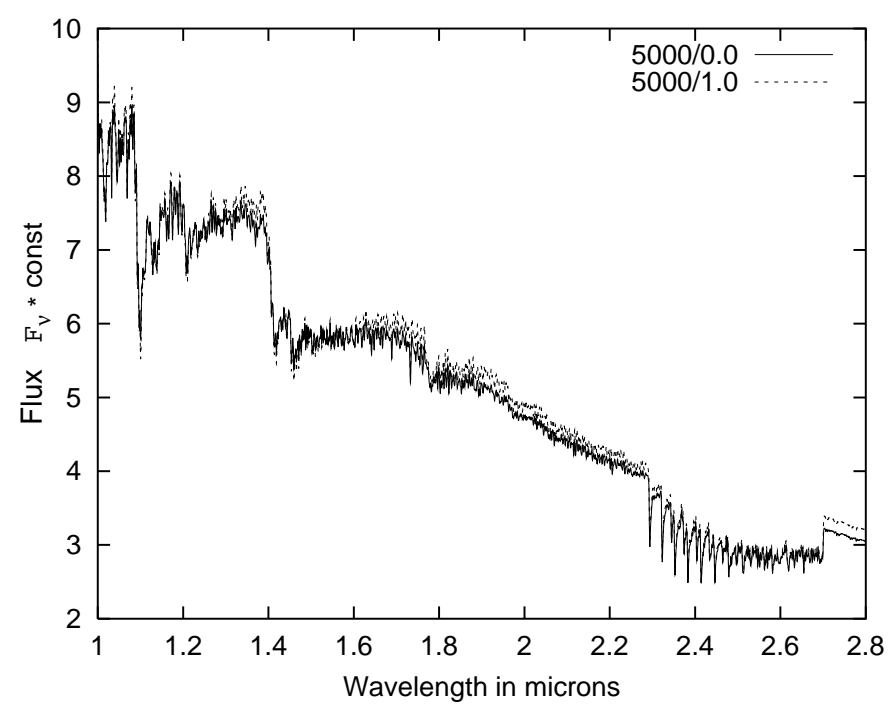

Fig. 3. Dependence of the model spectrum on $\log g$.

(Pavlenko et al. 2000). Thus it is not surprising that the optical spectra which match Sakurai's Object respond weakly to changes in the hydrogen abundance. This is in contrast to the behavior of models corresponding to the star a year earlier (Asplund et al. 1997). Similarly, the model IR spectra of Sakurai's Object for $T_{\text {eff }}=5000-6000 \mathrm{~K}$ depend weakly on $\log N(\mathrm{H})$ (Fig. 5). The magnitude of the change in the spectrum when $\log N(\mathrm{H})$ is changed from -2.42 (the Asplund et al. 1999 value for October 1996) to -0.97 (i.e, a change of $1.5 \mathrm{dex}$ ) is comparable (in a qualitative sense) to lowering $\log g$ from 1 to 0 (Fig. 3).

\subsection{Changes between 1997 April 21 and July 13}

Fits to the spectra of Sakurai's Object on April 21 and July 13 are shown in Figs. 6 and 7. The long wavelength portion of the $\mathrm{H}$ band is of special interest for the "carbon problem", because the strongest absorption bands of the $\mathrm{C}_{2}$ molecule, the Ballick-Ramsey bands, occur just longward of $1.768 \mu \mathrm{m}$. In the comparatively hot atmosphere of Sakurai's Object $\log N(\mathrm{C})>\log N(\mathrm{O})$ (Asplund et al. 1997, 1999) and the abundance of $\mathrm{C}_{2}$ depends mainly on the elemental abundance of carbon. Therefore, these bands may provide the most accurate determination of $\log N(\mathrm{C})$. The fits imply that the carbon abundance is in the range $\log N(\mathrm{C})=-1.3 \pm 0.2$. The most likely value is 0.3 dex higher than that found by Asplund et al. (1999). The accuracy of the determination of $\log N(\mathrm{C})$ is limited mainly by the quality of the molecular line list.

The effective temperatures that best fit the $1.0-2.0 \mu \mathrm{m} \mathrm{spec}-$ tra in 1997 April and July are $5500 \pm 200 \mathrm{~K}$ and $5250 \pm 200 \mathrm{~K}$, 


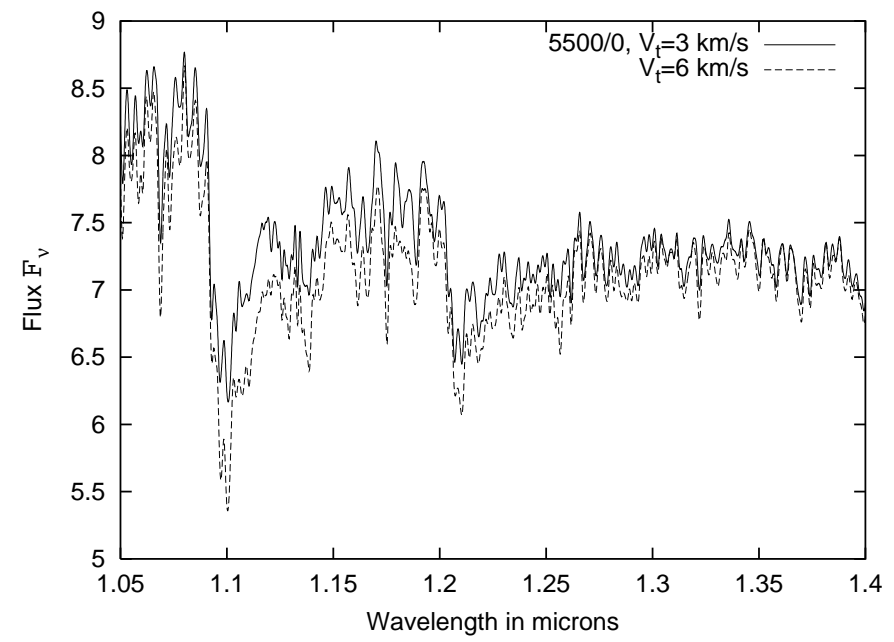

Fig. 4. Dependence of the model spectrum on $V_{\mathrm{t}}$.

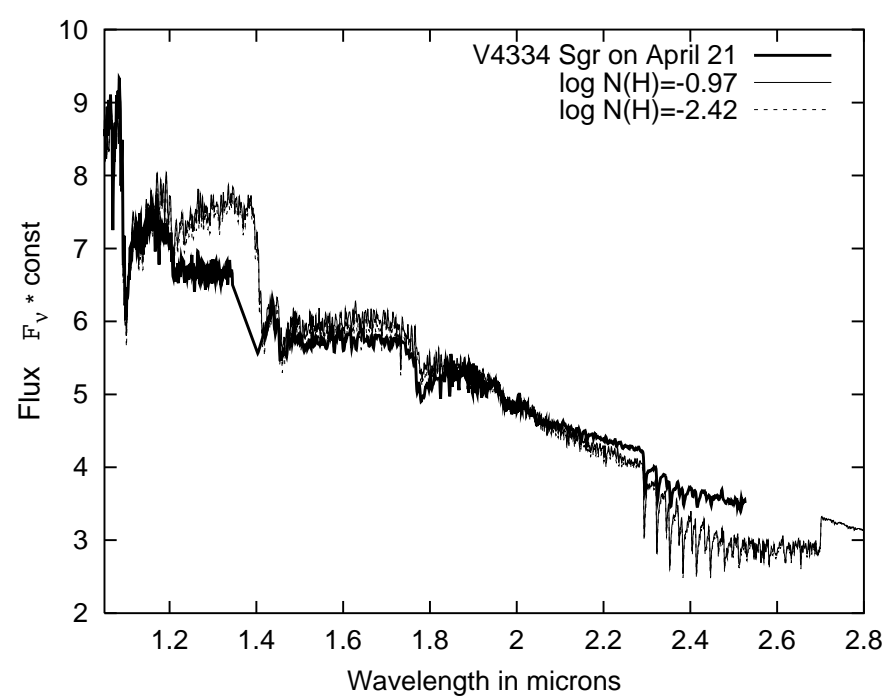

Fig. 5. Dependence of the model spectrum of Sakurai's Object on $\log N(\mathrm{H})$.

respectively, indicating that the cooling evidenced by the dramatic spectral changes seen between 1996 and 1997 (e.g., Geballe et al. 2002) continued in 1997. Our estimated uncertainties in the above temperatures are rather large, despite the comparatively good fits to the observed spectra, because of questions concerning abundances, non-sphericity effects, and dynamical phenomena, and because of contamination of the spectra by dust emission (see below).

\subsection{Hot dust}

Emission by dust is evident in the 1997 spectra by the mismatch between the synthetic and observed spectra longward of $2.0 \mu \mathrm{m}$ in Figs. 6 and 7. The difference between the observed and synthetic spectra is greater in the July spectrum, attesting to an increase in the amount of dust. The thermal emission from the dusty envelope overlaps the region of first overtone bands of ${ }^{12} \mathrm{CO}$ and ${ }^{13} \mathrm{CO}$ at $\lambda>2.3 \mu \mathrm{m}$. Usually these bands are used for determination of carbon abundances and isotopic ratios (cf. Lazarro et al. 1991). The reduced equivalent widths of the CO bands in July 1997 cannot be reasonably attributed to a large decrease in the oxygen abundance, because (1) this is unlikely to have occurred in three months and because the continuum shortward of the CO bands also shows an excess. We note that in fitting spectra, the most frequent situation is that the computed spectra have excess flux due to the deficit of known or hypothetical opacities. To fit the observed spectra, opacities in the model would need to be decreased at $\lambda>2 \mu \mathrm{m}$, an unrealistic possibility.

\section{Discussion}

Our analysis of the 1997 infrared spectra of Sakurai's Object strongly implies that dust was already present at that time. We note that Duerbeck (2002) did not find evidence for dust in the optical spectra from 1997. On the other hand, the fits by Pavlenko \& Duerbeck (2001) to the observed SEDs at optical wavelengths indicate that $E_{B-V}$ had increased by 0.6 (from 0.7 to 1.3) from April 1997 to August 1998. However, the August 1998 data were best fit using $T_{\text {eff }}=5250 \pm 200 \mathrm{~K}$, the same value as for July 1997 in this paper. Between June 1997 and August 1998 there were some variations in the photospheric radiaton, probably caused by mass losses events, evolution of the dusty envelope, and dynamical processes in the photosphere - envelope system (see light curve of Sakurai's Object in Duerbeck 2002). Nevertheless, $T_{\text {eff }}$ apparently remained nearly constant during this period.

In 1997, the year of maximum optical brightness of Sakurai's Object, the luminosity was still dominated by optical radiation. At that time "quasi-periodic fluctuations of increasing cycle length and amplitude were superimposed on the general brightness evolution" (Duerbeck 2002). In general, the effective temperature during such fluctuations does not need to follow changes of luminosity. In fact, it can be anti-correlated, since an increased radius can more than compensate for a lower $T_{\text {eff }}$. On the other hand, a change of radius can change the thermodynamical properties in the radiating region (i.e. photosphere). That may explain the similarity of $T_{\text {eff }}$ obtained in this paper for July 1997 and that found in August 1998 by Pavlenko \& Duerbeck (2001). The decreased optical brightness of Sakurai's Object in 1998 was mainly caused by development of the dust envelope (Kimeswenger 1999).

One question arises - were the optical and $2 \mu \mathrm{m}$ SED's being affected by the same dust in 1997-1998? The answer is probably yes. As mentioned earlier, the effective temperature remained constant during this time and thus cannot be the cause of the large change in $E_{B-V}$. This suggests that the cause of the increase in $E_{B-V}$ was newly formed dust. The new dust would be expected to have been close to the star and thus quite hot. Indeed the full 1-5 $\mu \mathrm{m}$ spectrum from 1998 (e.g., Geballe et al. 2002) shows that the excess peaked close to $3 \mu \mathrm{m}$, indicating a mean dust temperature close to $1000 \mathrm{~K}$ at that time. The dust must have been hotter (and closer) in 1997; this is supported by the data from 1997 (Eyres et al. 1998; Geballe et al. 2002) which show that the continuum flux density decreased monotonically with wavelength in the observed wavelength range, 1-4 $\mu \mathrm{m}$, at that time. Comparison of the 1997 and 1998 SEDs 

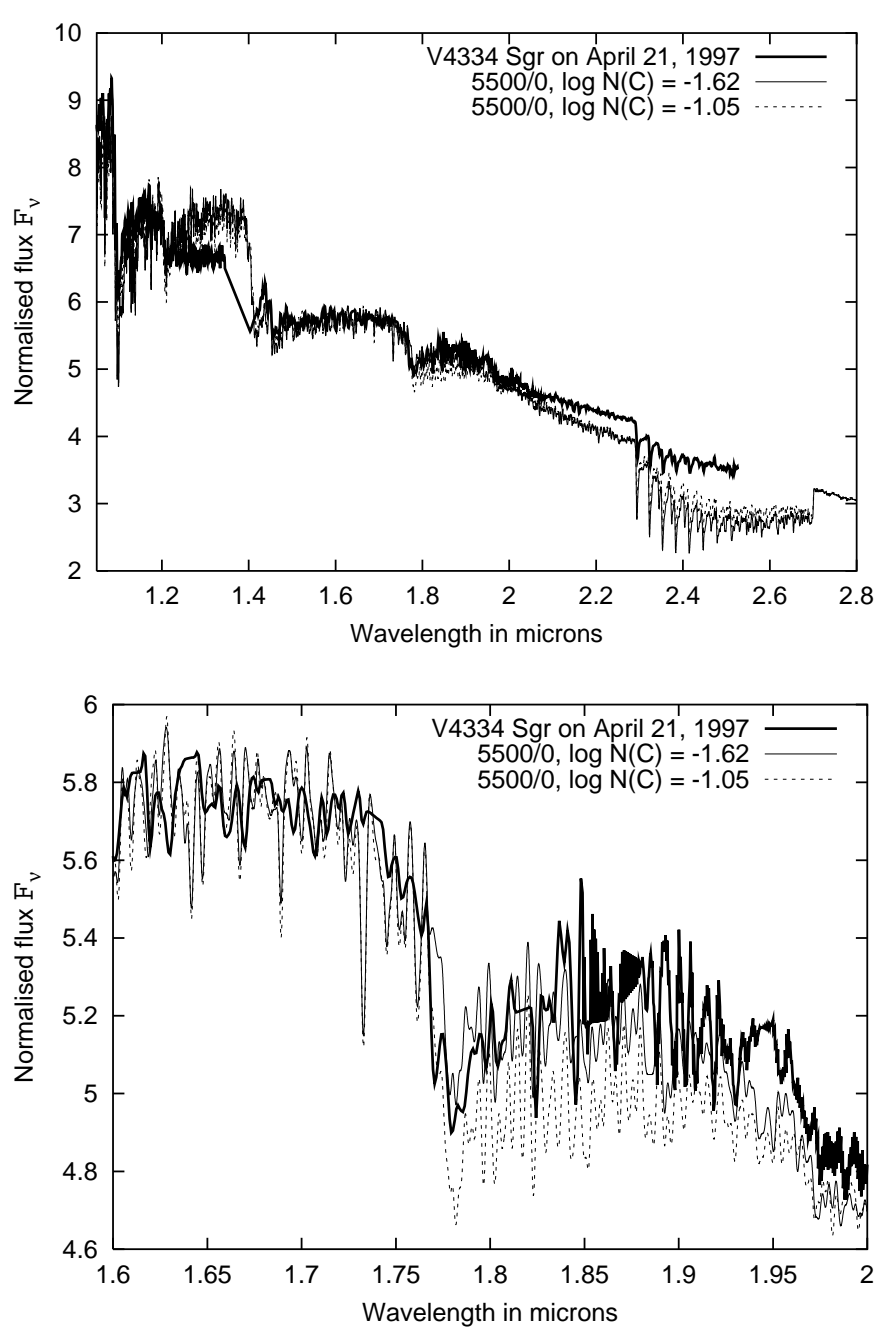

Fig. 6. Top: fits to observed spectrum of of Sakurai's Object on 1997 April 21. Bottom: details of the fits at 1.6-2.0 $\mu \mathrm{m}$; much of the structure at $1.82-1.95 \mu \mathrm{m}$ in the observed spectrum is due to incomplete removal of telluric absorption features. Synthetic spectra were computed for a microturbulent velocity of $6 \mathrm{~km} \mathrm{~s}^{-1}$.

also show that much less dust was present in 1997. Thus we confirm that the first appearance of dust occured in 1997 and that the amount of dust increased through summer 1998, prior to its becoming totally dominant in the latter part of 1998 and since then.

Acknowledgements. We thank the staff of the Joint Astronomy Centre for assistance in obtaining the spectra and the VALD database team for its helpful assistance. Partial financial support for YVP was provided by a Small Research Grant from the American Astronomical Society. TRG's research is supported by the Gemini Observatory, which is operated by the Association of Universities for Research in Astronomy, Inc., on behalf of the international Gemini partnership of Argentina, Australia, Brazil, Canada, Chile, the United Kingdom, and the United States of America. We thank the referee, M. Asplund for several helpful suggestions.
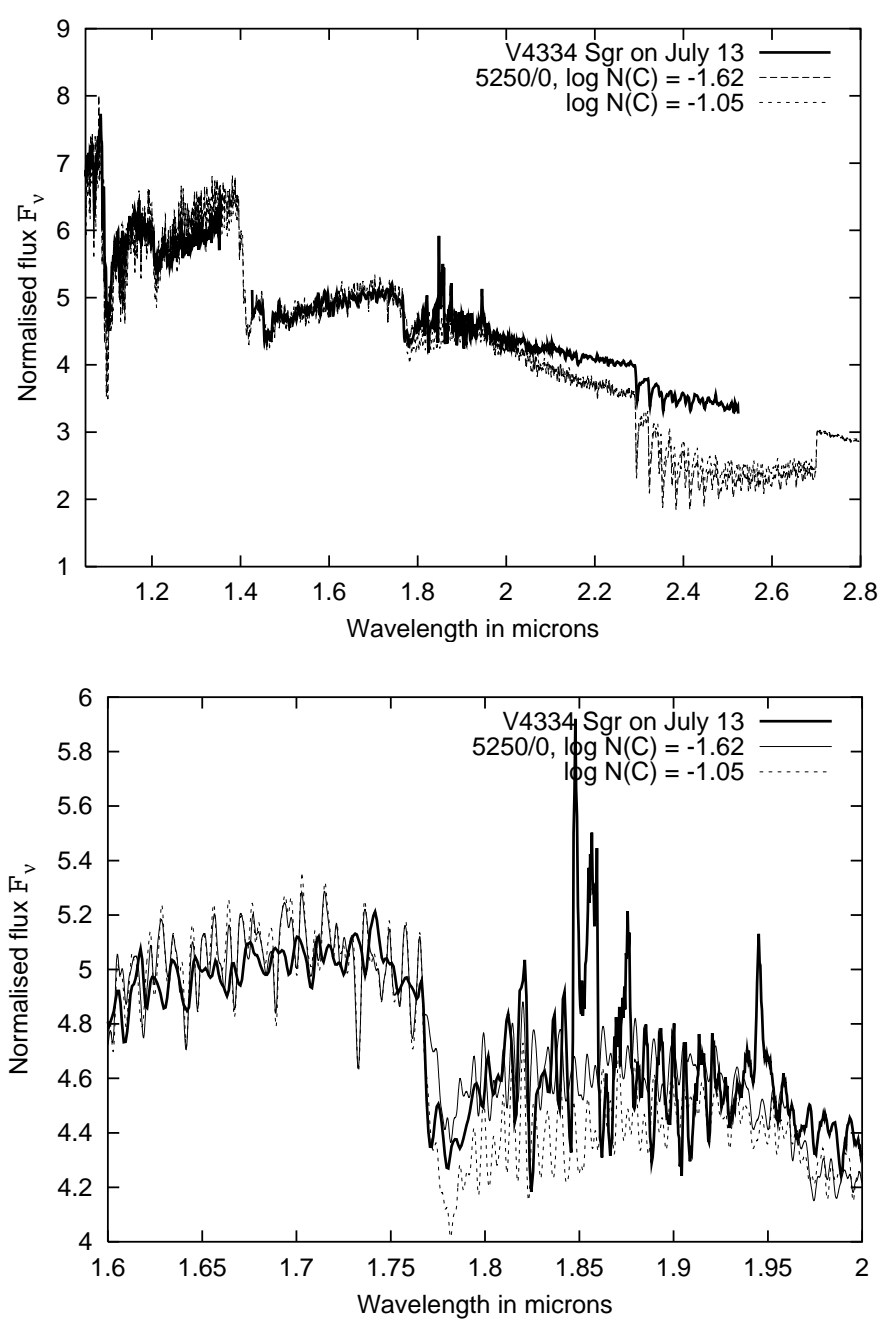

Fig. 7. Top: fits to observed spectrum of of Sakurai's Object on 1997 July 13. Bottom: details of the fit at 1.6-2.0 $\mu \mathrm{m}$. Synthetic spectra were computed for a microturbulent velocity of $6 \mathrm{~km} \mathrm{~s}^{-1}$.

\section{References}

Asplund, M., Gustafsson, B., Lambert, D. L., \& Rao, N. K. 1997, A\&A, 321, L17

Asplund, M., Gustafsson, B., Lambert, D. L., \& Kameswara Rao, N. 2000, A\&A, 353, 287

Asplund, M., Lambert, D. L., Kipper, T., Pollacco, D., \& Shetrone, M. D. 1999, A\&A, 343, 507

Duerbeck, H. W., \& Benetti, S. 1996, ApJ, 468, L111

Duerbeck, H. W. 2002, Astrophys. Space Sci., in press

Eyres, S. P. S., Evans, A., Geballe, T. R., Salama, A., \& Smalley, B. 1998, MNRAS, 298, L37

Geballe, T. G., Evans, A., Smalley, B., \& Eyres, S. P. S. 2002, Astrophys. Space Sci., in press

Kamath, U. S., \& Ashok, N. M. 1999, MNRAS, 302, 512

Kerber, F., Palsa, R., Köppen, J., Blöcker, T., \& Rosa, M. R. 2000, ESO Messenger, 101, 27

Kimeswenger, S., Gratl, H., Kerber, F., et al. 1997, IAU Circ., 6608

Kipper, T., \& Klochkova, V. 1997, A\&A, 324, L65

Kupka, F., Piskunov, N., Ryabchikova, T. A., Stempels, H. C., \& Weiss, W. W. 1999, A\&AS, 138, 119

Kurucz, R. L. 1993, CD-ROMs (Cambridge, Harvard Univ.)

Kurucz, R. L. 1999, http://www. cfa5 .harvard.edu 
Lazaro, C., Lynas-Gray, A. E., Clegg, R. E. S., Mountain, C. M., \& Zadrozny, A. 1991, MNRAS, 249, 62

Liller, W., Janson, M., Duerbeck, H. W., \& van Genderen, A. M. 1998, IAU Circ., 6825

Myerscough, V. P., \& McDowell, M. R. C. 1966, MNRAS, 132, 457

Nakano, S., Sakurai, Y., et al. 1996, IAU Circ., 6322

Pavlenko, Y. V., \& Yakovina, L. A. 1994, Astron. Rep., 38, 768

Pavlenko, Ya. V. 1997, ApSS, 253, 43

Pavlenko, Ya. V. 1999, Astron. Rep., 43, 94

Pavlenko, Ya. V., \& Duerbeck, H. W. 2001, A\&A, 367, 933

Pavlenko, Ya. V., \& Yakovina, L. A. 2000, Astron. Rep., 44, 209
Pavlenko, Ya. V. 2000, Astron. Rep., 44, 219

Pavlenko, Ya. V., Yakovina, L. A., \& Duerbeck, H. W. 2000, A\&A, 354, 229

Pavlenko, Ya. V. 2002, Astron. Rep., submitted

Pollacco, D. 1999, MNRAS, 304, 127

Seaton, M. J. 1992, RMAA, 23, 180

Sneden, C., Johnson, H., \& Krupp, B. 1976, ApJ, 204, 281

Unsold, A. 1955, Physik der Sternatmospheren, 2nd ed. (Springer, Berlin) 